\title{
LES MODÈLES ÉCONOMIQUES DE LA PRESSE Le lecteur comme fin en soi... ou comme cible pour les annonceurs?
}

\author{
Patrick Le Floch ${ }^{1}$
}

\section{Introduction : les fondements de l'économie de la presse}

Tout éditeur de presse connaît la complexité de l'économie du bien dont il doit assurer la gestion ${ }^{2}$. Le modèle économique d'un journal est en effet fondé sur le triptyque éditeur/lecteur/annonceur ${ }^{3}$. Au moment du lancement d'un titre, tout éditeur est confronté à un

1 Maître de conférences en économie, Institut d'Études Politiques de Rennes / CREREG.

2 Cette complexité n'est pas l'apanage de la presse. On la retrouve pour tous les biens dont les sources de recettes sont multiples (télévision, Internet, etc.).

3 Voir pour plus de détails P. LE FLOCH \& N. SonNAC, Économie de la presse, Paris, La Découverte, 2000. Ne sera pas évoqué dans cette contribution le cas assez rare des éditeurs qui ont décidé de rejeter les ressources publicitaires. V. Norris ("Mad Economics : an analysis of an adlkess magazine", Journalism of Communication, 1984, pp. 44-51) évoque le cas du magazine Mad aux États-Unis. En France, il est possible de citer Le Canard Enchaîné et Charlie Hebdo. Ces exemples ne seront pas analysés car ils ne correspondent pas à la norme des titres existant. Il convient simplement de retenir qu'il peut être possible de survivre ainsi.

Recherches en communication, $\mathrm{n}^{\circ} 21$ (2004). 
choix qui repose sur une combinaison a priori simple mais qui conduit à des implications stratégiques extrêmement fortes. Ses choix vont le conduire à proposer un produit rédactionnel destiné à un public donné. L'éditeur aura ensuite la possibilité de vendre son public aux annonceurs. La presse peut ainsi être un support publicitaire qui retient l'attention des annonceurs dans la mesure où ceux-ci sont en permanence à la recherche des techniques publicitaires susceptibles de valoriser les messages qu'ils désirent adresser à leurs consommateurs potentiels.

De façon générale, l'équilibre économique va principalement dépendre des orientations stratégiques qui seront privilégiées par un éditeur ${ }^{1}$. Tout un continuum de choix s'offrent à lui. Il pourra par exemple rechercher un contenu s'adressant à tous et assurant ainsi un niveau de diffusion très large. Sa politique de prix devra être en adéquation avec l'objectif recherché. La logique de la stratégie suivie est alors d'avoir un contenu généraliste et attractif, un prix de vente faible, une diffusion et une audience larges qui vont faire du titre un support incontournable pour les annonceurs. C'est dans les grands traits l'orientation suivie par les grands titres de presse magazine télévisée. A l'opposé de cette vision, l'éditeur pourra au contraire déterminer une cible plus restreinte qui est recherchée par les annonceurs. Il définira ensuite le contenu éditorial adapté à la cible recherchée. Les annonceurs viendront naturellement sur ce titre qui offre une cible spécifique non couverte par les autres titres. C'est une logique que l'on retrouve aussi bien sur certains marchés de niche de presse magazine ou sur le marché de la presse quotidienne gratuite rédactionnelle ${ }^{2}$.

La compréhension des interactions, qui naissent de la relation trilatérale entre l'éditeur, le lecteur et l'annonceur et qui permettent d'orienter les stratégies suivies par les éditeurs, nécessite de revenir sur les principales caractéristiques économiques de la presse en tant que bien. Une distinction sera ensuite opérée afin de distinguer la presse quotidienne de la presse magazine. L'existence d'une subvention croisée du marché de la publicité vers le marché de la

1 Voir N. SonNaC, "Lecteurs et publicité : l'incidence du comportement des lecteurs dans les stratégies des entreprises de presse écrite", Journée d'étude "Le lecteur", Institut d'Études Politiques de Rennes, 5 décembre 2002.

2 L'essentiel de la réussite de l'implantation de la presse quotidienne gratuite rédactionnelle provient du succès qu'elle rencontre auprès des lecteurs de moins de 35 ans qui ne sont habituellement pas des lecteurs de presse quotidienne payante. 
diffusion permettra de comprendre la spécificité des modèles économiques de la presse quotidienne. Nous verrons ensuite en quoi l'hétérogénéité des coûts de la presse a des implications fortes dans les stratégies suivies par les différents éditeurs. Le rôle de la publicité sera ensuite abordé tant sous l'angle de la concentration des marchés et de l'incidence qu'elle peut avoir en termes de contenus que sous l'angle de l'aversion potentielle que les lecteurs peuvent avoir vis-àvis d'elle.

\section{La presse : un bien économique complexe}

Les économistes font habituellement référence aux concepts de bien privé et de bien public pour établir une typologie des différents biens et services. Cette opposition bien privé/bien public leur permet ensuite de déterminer les conditions optimales de production de ces différents biens, l'objectif ultime étant de diagnostiquer si l'intervention de l'Etat ou d'une agence de régulation favorise l'efficacité économique. De nombreux travaux théoriques ont ainsi montré que le marché n'était pas la procédure optimale pour assurer la production de biens publics ${ }^{1}$. En l'absence de toute réglementation, le niveau de production des firmes privées est inférieur à la demande socialement optimale. Des besoins sont donc insatisfaits.

Dans son article fondateur, Samuelson ${ }^{2}$ rappelle les propriétés essentielles permettant de définir un bien collectif : la non-exclusion et la non-rivalité3. Dans ce cas de figure, "il est coûteux et nonsouhaitable d'exclure un individu de son utilisation, parce que les coût marginaux d' un individu utilisant un bien collectif sont nuls". Si l'information peut apparaître, lorsqu'elle n'est pas stratégique, comme un bien collectif pur, les titres de presse ont, quant à eux, des caractéristiques différentes. La condition de non-rivalité n'est en effet pas respectée. Le support papier rend le bien privatif : un individu achetant un exemplaire diminuera la quantité de journaux disponibles

I J.J. LAFFONT, Fondements de l'économie publique, vol. 1, Paris, Economica, 1988.

2 P.A. SAMUELSON, "The Pure Theory of Public Expenditure", Review of Economics and Statistics, 1954, pp. 387-389.

3 Pour une discussion plus approfondie sur ce point, voir P. LE FLOCH, Économie de la presse quotidienne régionale: déterminants et conséquences de la concentration, Paris, L'Harmattan/Syndicat de la Presse quotidienne régionale, 1997, pp. 30 et suivantes. 
aux autres individus. La rivalité de consommation a ainsi une implication forte pour les éditeurs de presse qui devront accepter de supporter des taux d'invendus non nuls, c'est-à-dire d'avoir un tirage supérieur au niveau de la demandel. Elle découle de l'existence d'un coût marginal de distribution qui n'est pas nul. Le point important est en l'occurrence que la rivalité de consommation est une des caractéristiques intrinsèques du support. Autrement dit, la nature du bien "information" est modifiée par le support sur lequel elle s'inscrit.

La complexité de la presse en tant que bien est encore accrue lorsque l'on considère la publicité. L'introduction de la publicité dans le journal conduit à un renforcement de la nature privative du bien : l'audience vendue aux annonceurs est un bien privé ${ }^{2}$ Cependant, comme le note parfaitement Kaldor ${ }^{3}$, la nature de la publicité en tant que bien est, elle aussi, ambiguë. La publicité est intrinsèquement persuasive : elle propose ainsi un contenu subjectif visant à influencer les consommateurs. Mais de nombreux travaux théoriques et empiriques insistent aussi sur le rôle informatif de la publicitét $:$ celleci peut en effet remplir une fonction de communication sociale en permettant un meilleur fonctionnement des marchés, tout particulièrement concernant les marchés de l'occasion. Dans cette perspective, la publicité partage (en partie) les spécificités propres au bien collectif.

$\mathrm{Au}$ total, les éditeurs de presse produisent des biens privés revêtant certaines caractéristiques de bien collectif. Il convient cependant de noter que la perception de l'information comme bien collectif ne donne pas lieu à une conception universelle. Si des

1 On peut noter qu'une offre supérieure à la demande n'implique pas nécessairement que la totalité de la demande soit satisfaite. Les éditeurs sont en effet confrontés au problème crucial du réglage des ventes. Malgré les invendus, des manques à la vente sont possibles dans tous les points de vente où le taux d'invendus est nul. Les services de vente cherchent donc à arbitrer entre la réduction du taux d'invendus (dans la mesure où ces invendus sont une source de coût) et la minimisation du nombre de points de vente avec un taux d'invendus nul...

2 Rappelons que les annonceurs ne sont pas seulement intéressés par les acheteurs directs du titre (i.e. les chiffres de diffusion), mais par le nombre de lecteurs : 1'audience correspond donc à la diffusion multipliée par le taux de circulation (nombre de lecteurs par exemplaire).

3 N. KALDOR, "The Economics Aspects of Advertising", Review of Economic Studies, 1950, pp. 1-27.

4 Voir P. NeLSON, "Advertising as Information", Journal of Political Economy, 1974 , pp. 729-754 et l'Avis du Conseil de la concurrence relatif au marché de la publicité en France (1987). 
réglementations existent dans quasiment tous les pays afin d'éviter une concentration excessive de la propriété des médias, des différences majeures existent en ce qui concerne la presse. A l'opposé de la conception anglo-saxonne qui accepte l'idée que la presse est un bien comme les autres, la France a de son côté considéré que les mécanismes du marché n'étaient pas idéaux pour assurer une production optimale et pluraliste ${ }^{1}$. C'est la raison pour laquelle l'intervention de l'État a été forte et s'est concrétisée à la fois par des réglementations et des aides à la presse (directes et indirectes) après la Seconde Guerre Mondiale.

\section{Subvention croisée et presse quotidienne}

C'est dans cet environnement particulier, lié au double marché de la presse (information et publicité) et à la nature des règles édictées par l'État, que les éditeurs vont choisir leur modèle économique. Ce choix aura des conséquences fortes sur l'équilibre financier du titre. Dans le cas d'une gratuité du titre, l'équilibre sera uniquement dépendant des recettes de publicité (qu'il s'agisse de publicité commerciale ou de petites annonces). Dans le cas de la presse quotidienne, le modèle économique reprend en partie la logique du modèle de la gratuité. En raison d'une option tarifaire consistant à fixer un prix de vente inférieur au coût moyen de production, la recherche de l'équilibre économique passe par la nécessité pour les titres de faire appel aux annonceurs. Plus la part des recettes publicitaires est forte relativement au chiffre d'affaire total du journal, plus le journal subira de plein fouet les retournements conjoncturels du marché publicitaire.

Le modèle économique de la presse magazine diffère substantiellement des modèles précédents ${ }^{2}$. Plusieurs travaux américains ont montré que la publicité ne "subventionnait" pas le titre via un prix de vente inférieur au coût de production ${ }^{3}$. Il semblerait en

I Il convient néanmoins de noter que les différentes réglementations et aides à la presse n'ont pas réussi à empêcher la tendance commune, observée dans tous les pays, à la concentration de la presse : le système français n'a, pour le moins, pas été efficient.

2 Sauf dans le cas de la presse magazine gratuite dite "consumer magazines".

3 V. NoRris, "Consumer Magazines and the Mythical Advertising Subsidy", Journalism Quarterly, 1982, pp. 205-211 ; L. SOLEY and R. KRISHNAN, "Does 
effet selon Kalita et Ducoffe que les recettes de vente permettent de couvrir les coûts rédactionnels de production, les titres à faible diffusion ayant tendance à privilégier des prix de vente élevés.

Le modèle économique de la presse quotidienne, aussi bien nationale que locale, repose au contraire sur l'existence d'une subvention croisée de la publicité vers le lecteur. Cette spécificité permet de comprendre les implications de ce modèle économique en termes de diffusion.

Tout d'abord, la recherche de la croissance de la diffusion pourra entraîner un journal, sinon à la faillite, tout au moins à une précarisation accrue de son équilibre financier. Un quotidien est en effet un produit joint offrant à la fois de l'information et de la publicité à ses lecteurs. Dans le cas de la presse quotidienne locale, les études empiriques montrent que ce produit est en adéquation avec les attentes du lectorat qui recherchent bien évidemment des informations rédactionnelles, mais aussi de la publicité (par exemple, les petites annonces) ${ }^{1}$.

Face à un événement particulier, le journal pourra accroître son tirage à la fois pour satisfaire une demande supplémentaire, mais aussi et surtout pour permettre à ses lecteurs réguliers de pouvoir se procurer leur titre comme pour tous les autres jours de la semaine. Mais, le plus souvent, les événements particuliers n'ont pas de répercussion durable sur les ventes. Aussi paradoxal que le constat puisse dans un premier temps paraître, une forte augmentation temporaire des ventes va dégrader la profitabilité des journaux. Le déficit d'exploitation, correspondant à l'écart entre le prix de vente et le coût moyen de production, va s'accroître dans les mêmes proportions que la hausse de la diffusion. Ce déficit pourrait théoriquement être réduit si le journal pouvait accroître son prix de vente ou s'il réussissait à réduire son coût moyen de production. Or

\footnotetext{
Advertising Subsidize Consumer Magazine Prices ?', Journal of Advertising, 1987, pp. 4-9 ; J.K. KALITA and R.H. DuCOFFE, A Simultaneus-equation Analysis of Pricing, Circulation and Advertising Revenue for Leading Consumer Magazines", Journal of Media Economics, 1995, pp. 1-16.

1 Rosse \& Dertouzos (J.N. Rosse and J.N. DeRTouzos, "Economic issues in mass communication industries", pp. 40-192, in Proceedings of the Symposium on Media Concentration, vol. 1, Bureau of Competition, Federal Trade Commission, 1978) et Lorimor (E.S. LORIMOR, "Classified Advertising : a Neglected Medium", Journal of Advertising, 1977, pp. 17-25) ont montré, dans le cas de la presse quotidienne américaine, que les lecteurs souhaitaient trouver dans leurs quotidiens de la publicité et particulièrement des petites annonces.
} 
ces hypothèses sont relativement irréalistes. Le journal ne modifie en effet pas son prix de vente en fonction d'une actualité imprévue ${ }^{1}$. En ce qui concerne maintenant le niveau des coûts moyens de production, les journaux opèrent en situation de rendements d'échelle constants ${ }^{2}$ : dans ces conditions de production, une hausse de la diffusion ne permettra donc pas de réduire significativement le niveau des coûts moyens. Seules des recettes publicitaires supplémentaires pourraient compenser le déficit d'exploitation. Mais, là encore, comme il avait déjà été possible de souligner la rigidité du prix de vente, on peut remarquer que les tarifs publicitaires sont fixés plusieurs mois à l'avance. La viscosité des tarifs publicitaires s'explique par le fait que les tarifs publicitaires se fondent sur les chiffres de diffusion et d'audience passés. Il en découle qu'un événement exceptionnel donnera lieu, au mieux, à une hausse différée des tarifs publicitaires si la hausse de diffusion s'avère être permanente... McChesney ${ }^{3}$ a ainsi montré que la recherche de scoop (Affaire du Watergate) n'avait eu aucun impact sur les ventes du Washington Post. Sur une base purement économique, il n'y a donc pas d'incitation particulière à rechercher une hausse de la diffusion. Ce constat n'est pas propre à la presse américaine: il est en effet uniquement induit par la caractéristique économique spécifique de la presse quotidienne d'avoir un prix de vente inférieur au coût moyen de production. C'est la raison pour laquelle Guéry ${ }^{4}$ rappelle que "gagner des lecteurs peut conduire à la faillite".

1 En revanche, dans le cas d'une information régulièrement plus dense ou d'une édition donnant lieu à la distribution de suppléments, le journal a la possibilité de modifier son prix de vente selon les jours de parution : le quotidien sportif L'Equipe est ainsi vendu plus cher le lundi et le samedi que les autres jours de la semaine.

2 Il faudrait que la hausse de la diffusion soit suffisamment forte pour que le journal réussisse à réduire mécaniquement son coût moyen de production, ce qui est théoriquement envisageable en raison de la forte proportion de coûts fixes dans le coût total. Mais, il ne faut pas oublier que la couverture d'un événement exceptionnel peut aussi donner lieu à des coûts supplémentaires pour un journal (hausse de la pagination, coût supplémentaire de collecte de l'information par la mobilisation d'une équipe journalistique renforcée...).

3 F.S. MCCHESNEY, "Sensationalism, Newspaper Profits and the Marginal Value of Watergate", Economic Inquiry, 1987, pp. 135-144.

4 L. GuÉRY, La presse régionale et locale, Paris, CFPJ, 1992. 


\section{Hétérogénéité des coûts et tailles minimales efficientes}

Dans la vision anglo-saxonne qui appréhende le journal comme un bien comme les autres, McChesney ${ }^{1}$ va jusqu'à considérer que l'information rédactionnelle peut être assimilée à un produit d'appel, technique de marketing très largement utilisée dans d'autres secteurs économiques. Pour attirer les consommateurs dans les grandes surfaces, les enseignes n'hésitent pas à offrir des produits d'appel à des prix inférieurs à leurs coûts de production. De la même façon, le journal est vendu à un prix inférieur à son coût de production afin d'atteindre une cible suffisante à laquelle s'adressent les messages publicitaires.

Cette stratégie, avérée dans le cas de la presse quotidienne, peut se comprendre au regard de la structure et du niveau des coûts supportés par les différentes familles de presse. Quelle que soit la périodicité du titre, l'éditeur est en effet obligé d'atteindre le niveau de diffusion à partir duquel il ne bénéficie plus du phénomène des économies d'échelle. Ce phénomène est observable dans toutes les industries dans lesquelles (mais ce n'est pas une condition unique) existent des coûts fixes importants. Il en découle alors; mécaniquement, que ceteris paribus une hausse de la diffusion permet d'amortir sur un plus grand nombre d'exemplaires les coûts fixes engagés dans le processus productif : le coût moyen est donc décroissant.

Un constat simple, qui n'est pas propre aux entreprises de presse, peut être fait à ce niveau : la taille minimale efficiente (i.e. le seuil à partir duquel le journal opère en situation de rendements d'échelle constants) dépendra de la part des coûts fixes dans le coût total. Si les éditeurs ont évidemment des marges de manœuvre à ce niveau, il n'en demeure pas moins que de grandes différences existent selon les familles de presse. Le tableau 1 montre ainsi que la part des salaires et des charges sociales est très significativement supérieure dans la presse quotidienne que dans la presse magazine. Les éditeurs de quotidiens sont stratégiquement contraints d'intégrer toutes les activités qu'ils considèrent comme étant nécessaires à la maîtrise de leur environnement (équipe rédactionnelle étoffée, maîtrise d'un parc de rotatives, etc.). A l'inverse, les groupes de presse magazine

1 F.S. MCCHESNEY, op. cit. 
recourent de façon systématique à l'externalisation ${ }^{1}$. La sous-traitance leur permet de rendre variables des coûts qui sont fixes dans la presse quotidienne. Les tailles minimales efficientes des titres de presse magazine peuvent donc être réduites. L'implication est que l'éditeur de presse magazine pourra ajuster assez facilement son niveau de coût à la cible qu'il recherche. Il lui suffira, par exemple, d'allonger sa périodicité pour rendre viable son titre. Les barrières à l'entrée sont réduites, ce qui permet de comprendre pourquoi il est relativement aisé de créer de nouveaux magazines ${ }^{2}$. Il n'en demeure pas moins que, si le lancement de titre est facilité, seuls les titres satisfaisant un public donné survivront.

Tableau 1 - Structure de coûts selon les familles de presse en France

\begin{tabular}{|c|c|c|c|}
\hline & $\begin{array}{c}\text { Salaires et } \\
\text { charges sociales }\end{array}$ & $\begin{array}{c}\text { Matières } \\
\text { premières }\end{array}$ & $\begin{array}{c}\text { Autres achats } \\
\text { et achats externes }\end{array}$ \\
\hline PQR & 38,8 & 10,4 & 44,9 \\
\hline PHR & 37,6 & 6,7 & 49,1 \\
\hline Presse magazine $^{2}$ & 20,7 & 7,1 & 60,3 \\
\hline
\end{tabular}

Les chiffres sont donnés en pourcentage du total des charges. ' : incluant presse magazine grand public et presse professionnelle. $\mathrm{PQR}$ : presse quotidienne régionale. PHR : presse hebdomadaire régionale. Source : La Loupe Financière (1999), France.

La recherche de l'équilibre financier du modèle économique de la presse quotidienne apparaît plus complexe qu'elle ne l'est dans le cas de la presse magazine. La nécessité d'internaliser les opérations de production vitales conduit les éditeurs à supporter des coûts élevés et, par conséquent, à rechercher une taille critique importante. Les éditeurs sont confrontés alors à un dilemme simple : soit ils font supporter aux consommateurs le coût de production du journal, soit ils font appel aux ressources publicitaires. Adopter la première alternative impliquerait pour le journal d'être confronté à une spirale de la diffusion négative ${ }^{3}$ : fixer un prix de vente élevé signifie que le

1 Pour plus de détails en presse quotidienne et en presse magazine, voir respectivement P. LE FLOCH, Économie de la presse quotidienne régionale, op. cit. et J.-M. Charon, La presse magazine, Paris, La Découverte, 2001.

2 Ces différences de coût ont des incidences en termes de profitabilité : mesurée au niveau de la marge d'exploitation, la profitabilité de la presse magazine française est de $8,8 \%$ sur la période $1995-1999$ contre $3,9 \%$ pour la presse quotidienne régionale et $-0,4 \%$ pour la presse hebdomadaire régionale.

3 Furhoff (L. FurHOFF, "Some Reflections on Newspaper Concentration", Scandinavian Economic History Review, 1973, pp. 1-27.1973) et Gustafsson 
journal découragera une partie des acheteurs ; le journal verra alors son coût moyen de production s'accroître. Deux solutions s'offrent à lui : accrôtre de nouveau son prix... ou diminuer ses coûts fixes. Mais dans les deux cas, cette stratégie poussera le journal à la faillite. Il n'est donc pas surprenant de comprendre pourquoi les éditeurs ont opté pour la stratégie consistant à faire appel aux recettes publicitaires afin de pouvoir proposer un contenu rédactionnel d'une qualité donnée à un grand nombre de lecteurs.

Tableau 2 - Répartition des recettes (en \%) selon les familles de presse

\begin{tabular}{|c|c|c|c|}
\hline & 1985 & 1990 & 2000 \\
\hline PQN & 1,25 & 1,7 & 1,9 \\
\hline Ventes au numéro & 43,2 & 33,9 & 30,0 \\
\hline Abonnement & 14,4 & 14,3 & 17,6 \\
\hline Publicité commerciale & 30,4 & 32,7 & 40,6 \\
\hline Annonces & 12,8 & 19,0 & 11,8 \\
\hline PQR et PHR & 1,75 & 2,3 & $\mathbf{3 , 0}$ \\
\hline Ventes au numéro & 46,9 & 42,0 & 39,5 \\
\hline Abonnement & 13,7 & 12,5 & 16,2 \\
\hline Publicité commerciale & 29,1 & 30,3 & 28,7 \\
\hline Annonces & 10,3 & 15,6 & 15,2 \\
\hline $\begin{array}{l}\text { Presse magazine grand } \\
\text { public }\end{array}$ & 2,3 & $\mathbf{3 , 1}$ & 4,1 \\
\hline Ventes au numéro & 56,1 & 52,3 & 48,6 \\
\hline Abonnement & 14,3 & 16,3 & 19,6 \\
\hline Publicité commerciale & 27,8 & 28,1 & 29,0 \\
\hline Annonces & 1,7 & 2,9 & 2,9 \\
\hline $\begin{array}{l}\text { Presse magazine } \\
\text { professionnelle }\end{array}$ & 0,6 & 1,1 & 1,1 \\
\hline Ventes au numéro & 6,2 & 4,6 & 4,4 \\
\hline Abonnement & 39,1 & 30,6 & 40,7 \\
\hline Publicité commerciale & 46,9 & 50,0 & 43,3 \\
\hline Annonces & 17,8 & 13,0 & 11,5 \\
\hline
\end{tabular}

Le chiffre d'affaires en caractère gras est donné en milliards d'euros. $\mathrm{PQN}$ : presse quotidienne nationale. Source : DDM, France

Le tableau 2 confirme l'importance relative des recettes publicitaires dans la presse quotidienne. Dans le cas français, la part

(K.E. Gustafsson, "The Circulation Spiral and the Principle of Household Coverage", Scandinavian Economic History Review, 1978, pp. 1-14) ont décrit les conséquences de ce mécanisme dans le cas où deux journaux sont en situation de concurrence : la tendance à la monopolisation des marchés est souvent ce qui est observe. 
des recettes publicitaires est assez faible au regard des situations que l'on peut observer aux États-Unis, en Grande-Bretagne et dans les pays scandinaves où il n'est pas rare que les recettes publicitaires représentent jusqu'à $80 \%$ des recettes totales.

\section{Publicité et concentration}

Si la publicité exerce indéniablement un effet indirect positif sur la diffusion des supports en "subventionnant" le journal, elle soulève néanmoins quelques interrogations quant aux conséquences qu'elle peut avoir tant en termes de contenus que de concentration des marchés.

La recherche de recettes publicitaires peut, dans certains cas, apparaître comme une fin en soi. Si cette approche de la presse ne soulève aucun problème dans de nombreux pays, elle continue de poser question en France. Il convient à ce niveau de préciser un point important. Les logiques en ouvre dans la presse quotidienne et dans la presse magazine ne sont pas les mêmes. La presse magazine est un secteur économiquement très rentable où l'objectif de la majorité des groupes de presse est clairement affiché : la recherche de la rentabilité maximale. Dans cette perspective, toutes les stratégies sont défendables : un éditeur pourra ainsi commencer par s'intéresser à des annonceurs qui ne trouvent pas le bon support pour atteindre leur cible; s'il estime le marché viable, il proposera un contenu rédactionnel spécifique qui devra satisfaire les catégories socioprofessionnelles recherchées par les annonceurs. Les Editions Jalouse ont pu suivre cette stratégie pour certains de leurs titres. Le secteur de la presse magazine est devenue un secteur économique comme un autre'.

La question de l'influence de la publicité se pose en des termes un peu différents dans le cas des journaux d'informations politiques et générales. Les quotidiens ont adopté la stratégie d'un prix de vente faible $^{2}$. Les recettes publicitaires représentent un élément

1 Une remarque peut être faite à ce sujet concernant le système d'aides en France : si la presse magazine est un bien comme les autres, les aides publiques se justifientelles encore?

2 Le terme faible est à interpréter de façon relative. Dans le cas de la France, les quotidiens, particulièrement les nationaux, sont beaucoup plus chers que les quotidiens vendus dans d'autres pays (c'est d'ailleurs une raison souvent avancée 
complémentaire essentiel dans la recherche de l'équilibre économique. La première implication pour les journaux est alors de les rendre dépendants de la situation du marché publicitaire. Lorsque l'économie connaît une croissance économique inférieure à sa croissance potentielle, les entreprises cherchent à comprimer leurs coûts afin de limiter leurs pertes ou la dégradation de leurs profits. Les dépenses publicitaires sont à cet égard particulièrement volatiles dans la mesure où elles constituent un des premiers postes budgétaires sur lesquels les entreprises réalisent des économies. Le modèle économique des quotidiens sera par conséquent tributaire de cet aléa. Ce qui conduit les journaux à voir leur rentabilité surréagir par rapport au cycle économique.

L'incidence de la publicité est aussi visible dans le processus de concentration de la presse. Les annonceurs ont en effet tendance à donner une prime au leader : un journal dominant son marché aura une part du marché publicitaire plus que proportionnelle à la part qu'il détient sur le marché de la diffusion ${ }^{1}$. Le triptyque éditeur/lecteur/annonceur prend ici tout son sens. Compte tenu du choix initial d'un prix de vente subventionné, l'éditeur sera contraint d'être le leader sur son marché afin d'assurer la viabilité économique de son titre en captant les recettes publicitaires. On comprend alors la logique en œuvre dans le secteur de la presse quotidienne. Elle n'est pas nouvelle, loin de là. Le rédacteur en chef d'un quotidien américain décrivait déjà en 1881 l'orientation que devait suivre son journal : "The Star has no axe to grind, no candidate to elect, no party to serve. Its only interest is in the growth and prosperity of Kansas City and the proper administration of the city government" 2 . Le journal doit s'implanter sur une zone qu'il doit défendre pour assurer sa survie qui dépendra de son marché publicitaire, autrement dit de la santé économique de la zone qu'il couvre. La concentration des marchés locaux de la presse quotidienne dans les différents pays industrialisés s'explique pour une grande part par ce constat. Elle a

pour expliquer la faiblesse du taux de pénétration de la presse quotidienne nationale française). S'ils sont chers, ils bénéficient néanmoins d'une subvention croisée : un journal comme Le Monde devrait accroître son prix de vente de 40 à $50 \%$ s'il désirait couvrir ses coûts de production (hors publicité).

1 La théorie de la spirale de la diffusion de Gustaffson (op. cit.) se fonde sur ce constat pour expliquer la tendance à la concentration des marches de la presse.

2 Cité in J. Rogers, Newspaper Building. Application of efficiency to Editing, to Mechanical Production, to Circulation and Advertising, New York, Harper \& Brothers, 1918, p. 28. 
des incidences en termes de contenu. D'une part, elle conduit à la disparition des journaux d'opinion, tout particulièrement dans le cas où l'audience de ces journaux n'intéresse pas les annonceurs ${ }^{1}$. En 1951, Ray avait très bien résumé la logique en œuvre : il parlait de "middle of the road policy"... L'évolution du secteur de la presse quotidienne régionale depuis 1945 a confirmé cette tendance qui fait que, pour survivre, les journaux cessent d'être des journaux d'opinion et deviennent soit des journaux au contenu plus neutre, soit des journaux d'opinions ${ }^{2}$. D'autre part, la concentration des marchés réduit la qualité des journaux et exerce une influence négative sur le niveau de la diffusion ${ }^{3}$. Le manque de concurrence n'incite les rédactions ni à rechercher, ni à traiter l'information rapidement. L'aiguillon de la concurrence est pourtant un élément essentiel. On peut penser que l'excellente qualité de la presse en Bretagne (qui a, pour corollaire, un taux de pénétration très élevé dans un département comme le Finistère) est due à la concurrence existant entre OuestFrance et Le Télégramme.

La concentration, induite par les interactions entre les marchés de la diffusion et de la publicité, peut donc conduire à un appauvrissement relatif des contenus en termes de diversité. Il convient cependant de relativiser ce constat. D'une part, il est sans doute beaucoup plus vrai pour la presse quotidienne que pour la presse magazine qui se caractérise par une très grande diversité de titres proposée aux lecteurs ${ }^{4}$. D'autre part, les quotidiens se sont diversifiés au niveau du contenu (politique de suppléments) afin d'offrir de nouveaux espaces publicitaires aux annonceurs ${ }^{5}$.

1 En France, le quotidien $L ' H u m a n i t e ́$ est entré dans une spirale récessive depuis de nombreuses années : seuls des dons privés et des aides exceptionnelles de l'État lui permettent de continuer à vivre.

2 N. Schulz \& J. Weimann ("Competition of Newspapers and the Location of Political Parties", Public Choice, 1989, pp. 125-147) et J.J. Gabszewicz \& alii ("Press Advertising and the Ascent of Pensée Unique", European Economic Review, 2001, pp. 641-651) ont modélisé l'observation faite par Ray.

3 P. LE FLOCH, Economie de la presse quotidienne régionale, op. cit.

4 Dans le cas de la presse magazine française, on doit néanmoins rappeler que la différenciation des titres n'implique pas un marché parfaitement concurrentiel. Les six plus grands groupes de presse magazine représentent $70 \%$ du marché total.

5 La publicité fait aussi courir un risque en termes de contenu sous la forme d'articles de complaisance (une sorte de publi-rédactionnel) qui n'ont comme seule justification que de mettre en valeur l'espace publicitaire acheté par les annonceurs. Une autre forme de risque est celle de la censure ou le plus souvent de l'autocensure sur le plan rédactionnel. Un journaliste a-t-il la possibilité de s'exprimer librement et objectivement sur un support dans lequel le sujet de son article porte 


\section{Publicité et aversion des lecteurs}

La publicité occupe une place très importante dans l'économie de la presse. L'analyse détaillée du triptyque éditeur/lecteur/ annonceur montré même que les annonceurs bénéficient de la concentration des marchés de la diffusion. Reimer ${ }^{1}$ et Le Floch ${ }^{2}$ ont mesuré que, ceteris paribus, le coût de la publicité était plus faible pour un annonceur confronté à un monopoleur que lorsqu'il avait affaire à un duopole, ceci tenant principalement à la non duplication des coûts fixes dans le cas d'un monopole.

Au-delà des éléments évoqués dans les points précédents, il est important de rappeler que l'éditeur est contraint dans les choix qu'il peut faire en termes de publicité. En France, le versement des aides de l'État est conditionné par le respect par les titres d'une proportion maximale de publicité dans la pagination totale : la contrainte, qui n'est pas très forte, limite la surface publicitaire à deux tiers de la surface totale. Mais la contrainte la plus importante n'est pas à ce niveau : elle concerne la réaction des lecteurs à la publicité. Les lecteurs sont-ils publiphiles ou publiphobes?

Dans le cas d'une publicité considérée comme intrusive, le message publicitaire fait supporter une externalité négative au lecteur ${ }^{3}$. La complexité de la réaction des lecteurs s'explique dans la mesure où la publicité peut combiner à la fois des éléments objectifs (sources d'externalités positives) et subjectifs (sources d'externalités négatives).

De nombreuses études empiriques ont permis de tester l'hypothèse de l'aversion des individus à la publicité dans les différents médias ${ }^{4}$. S'il apparaît dans le cas de la télévision que les individus se déclarent publiphobes, la réaction est plus complexe

sur un annonceur important? Le meilleur moyen pour un journal de conserver son indépendance est certainement de diversifier ses recettes publicitaires.

1 E. REIMER, "The Effects of Monoôlization on Newspaper Advertising Rates", American Economist, 1992, pp. 65-70.

2 P. LE FLOCH, "Droits de propriété et espace publicitaire dans la presse quotidienne régionale", Revue d’Économie Industrielle, 1996, pp. 31-46.

3 L'intrusion de la publicité est particulièrement visible sur Internet (pop up...).

4 Pour une bibliographie détaillée, voir J.J. GabSzEwiCZ, D. LAuSSEL \& N. SonNAC, "Attitudes towards advertising and Price Competition in the Press Industry, Colloque Journées de Microéconomie Appliquée, Rennes, 2002. 
concernant la presse. Certains travaux américains ont en effet montré que la demande des journaux s'accroissait avec la publicité. Ce qui implique que les consommateurs peuvent apprécier la publicité.

Tableau 3 - Pourcentage de publiphobes dans la presse

\begin{tabular}{|l|c|c|}
\hline & Quotidiens & Magazines \\
\hline France & 51 & 50 \\
\hline Italie & 51 & 46 \\
\hline Espagne & 48 & 43 \\
\hline Allemagne & 54 & 43 \\
\hline Grande-Bretagne & 62 & 53 \\
\hline
\end{tabular}

Source : $C B$ News ${ }^{1}$.

Une enquête récente réalisée dans quinze pays européens par l'institut allemand Gfk montre que les consommateurs européens ne réagissent pas de la même manière face à la publicité ${ }^{2}$. Dans des pays comme l'Espagne et l'Allemagne, une large majorité de consommateurs (supérieure à $80 \%$ ) estiment que la place de la publicité est trop importante. L'aversion des consommateurs à la publicité peut s'expliquer en raison de la frustration qu'ils ressentent face à des produits qu'ils ne peuvent pas acheter. Le rejet de la publicité peut enfin s'expliquer par le refus des techniques publicitaires (coupure publicitaire dans les programmes télévisés, prospectus dans les boîtes aux lettres...). A l'opposé, les consommateurs britanniques et slovaques apprécient la publicité à plus de $70 \%$. En Grande-Bretagne, il ne semble pas exister de frein culturel à la publicité. Selon une autre enquête réalisée pour le Syndicat National de la Publicité Télévisée par IPSOS, 41\% des Français se déclarent publiphobes (pour l'ensemble de l'Europe, le pourcentage est de $39 \%)^{3}$. Mais, comme le rappelle fort justement Hervé Barbot, directeur général adjoint de IPSOS Médias, "c'est une étude d'opinion, pas de comportement": se déclarer publiphobe n'implique pas qu'on le soit réellement.

1 Musnik, "Le cœur de cible ne porte pas la pub dans son cœur", CB News, 11-17 octobre 1999.

2 Le Monde, 8 septembre 1998.

3 Sébastien LebourcQ, “Les Français publiphobes? ”, L'Express, 28 février 2002. 
Il en découle alors que tous les médias doivent tenir compte de cette réaction lorsqu'ils réfléchissent à la quantité "optimale" de publicité qu'ils désirent introduire dans leur support. Une forte aversion à la publicité des lecteurs pourrait en effet avoir des conséquences négatives en termes de diffusion. Cependant, force est de constater que peu d'éléments objectifs chiffrés permettent de soutenir l'hypothèse d'aversion ${ }^{1}$. Celle-ci est en revanche avérée sur les supports où les consommateurs ne peuvent que difficilement éviter le message publicitaire (télévision, Internet). Dans le cas de la presse magazine féminine (comme Elle par exemple), il semble que les lecteurs ne sont pas perturbés par la très forte pagination publicitaire. A partir du moment où le chemin de fer (i.e. la présentation du magazine et l'ordonnancement des rubriques) est stable, le lecteur ne perd pas de temps pour retrouver ses rubriques préférées. Autrement dit, c'est certainement la possibilité de "zapper" qui fait qu'il ne vit pas nécessairement mal la présence de la publicité dans son magazine.

\section{Conclusion}

Le triptyque éditeur/lecteur/annonceur est la base sur laquelle reposent les orientations définies par les éditeurs. Les stratégies polaires sont minoritaires. D'un côté, peu d'éditeurs rejettent d'emblée la ressource publicitaire. A l'opposé, les lancements de titre de presse gratuite montrent les difficultés que peuvent rencontrer ces supports. Les consumer magazines souffrent d'un manque de crédibilité pour attirer les annonceurs nécessaires à la rentabilité. La presse quotidienne gratuite rédactionnelle rencontre, quant à elle, un plus grand succès en touchant une cible habituellement non couverte par la presse quotidienne traditionnelle, mais souffre, tout particulièrement dans la période de lancement, de la dégradation du marché publicitaire (ce qui ne remet pas en cause que tel le concept spécifique que des groupes comme Metro et Schibsted ont élaboré).

Le triptyque conduit le plus souvent à l'adoption d'une stratégie mixte où les éditeurs se fondent à la fois sur les recettes de vente et de publicité. On a pu voir combien la logique économique de la presse

1 Sonnac ("Lecteurs et publicité...", op. cit.) note fort justement que peu d'études empiriques ont été publiées sur le sujet dans la mesure où ni les annonceurs ni les éditeurs n'ont intérêt à diffuser des résultats soutenant l'hypothèse de la publiphobie. 
magazine était radicalement différente de celle de la presse quotidienne. La presse magazine apparaît en moyenne, non seulement plus rentable, mais aussi moins sujette aux aléas conjoncturels rencontrés sur le marché publicitaire. Tous les modèles sont possibles en presse magazine (recettes fondées sur l'abonnement ou sur les ventes au numéro; recherche d'une cible intéressant les annonceurs et définition d'un contenu éditorial). La presse magazine est tout simplement un bien comme les autres.

En revanche, plusieurs interrogations peuvent se poser en ce qui concerne la presse quotidienne. En raison de ses caractéristiques économiques, la presse quotidienne a fait le choix d'un modèle fondé sur la publicité qui lui est nécessaire pour essayer de diffuser le plus largement possible. L'influence de la publicité y est donc très forte au niveau des contenus : les journaux proposent des suppléments thématiques; ils cherchent aussi à investir dans des matériels de production onéreux qui assurent une impression en quadrichromie. L'influence est aussi importante au niveau de l'évolution des structures de marché : la publicité renforce la tendance, induite par la structure des coûts, à la concentration des marchés. Celle-ci n'est évidemment pas sans influence sur le plan de la qualité de l'information. C'est pourquoi on peut se demander, par provocation si la différence entre la presse quotidienne traditionnelle et la presse quotidienne gratuite existe réellement. En effet, ces deux modèles reposent sur une base identique : une tarification du prix de vente inférieure au coût moyen de production. La presse quotidienne gratuite n'a fait que pousser à l'extrême la logique qui était en œuvre dans la presse quotidienne payante. En définitive, la question qu'il conviendrait de se poser est de savoir comment l'on définit la presse en tant que bien. Cette question est encore primordiale dans la mesure où, dans un pays comme la France, on a élaboré un système d'aides complexes à la presse qui reposait sur l'idée que la presse n'était pas un bien comme les autres. Si le secteur de la presse a évolué, le système d'aides ne devrait-il pas en faire autant? 